\title{
A Pathway in the Brainstem for Roll-Tilt of the Subjective Visual Vertical: Evidence from a Lesion-Behavior Mapping Study
}

\author{
Bernhard Baier, ${ }^{1}$ Frank Thömke, ${ }^{1}$ Janine Wilting, ${ }^{1}$ Caroline Heinze, ${ }^{1}$ Christian Geber, ${ }^{1}$ and Marianne Dieterich ${ }^{1,2}$ \\ ${ }^{1}$ Department of Neurology, University of Mainz, 55131 Mainz, Germany, and ${ }^{2}$ Department of Neurology and Integrated Center For Research and Treatment \\ of Vertigo Balance and Ocular Motor Disorders, Ludwig-Maximillians-University, 80539 Munich, Germany
}

\begin{abstract}
The perceived subjective visual vertical (SVV) is an important sign of a vestibular otolith tone imbalance in the roll plane. Previous studies suggested that unilateral pontomedullary brainstem lesions cause ipsiversive roll-tilt of SVV, whereas pontomesencephalic lesions cause contraversive roll-tilts of SVV. However, previous data were of limited quality and lacked a statistical approach. We therefore tested roll-tilt of the SVV in 79 human patients with acute unilateral brainstem lesions due to stroke by applying modern statistical lesionbehavior mapping analysis. Roll-tilt of the SVV was verified to be a brainstem sign, and for the first time it was confirmed statistically that lesions of the medial longitudinal fasciculus (MLF) and the medial vestibular nucleus are associated with ipsiversive tilt of the SVV, whereas contraversive tilts are associated with lesions affecting the rostral interstitial nucleus of the MLF, the superior cerebellar peduncle, the oculomotor nucleus, and the interstitial nucleus of Cajal. Thus, these structures constitute the anatomical pathway in the brainstem for verticality perception. Present data indicate that graviceptive otolith signals present a predominant role in the multisen-
\end{abstract} sory system of verticality perception.

\section{Introduction}

Ocular tilt reaction (OTR) consists of the triad skew deviation, head tilt, and ocular torsion. It is a clinical sign of a vestibular tone imbalance in the roll plane (Halmagyi et al., 1979). The roll-tilt of the subjective visual vertical (SVV) is considered to be the perceptual correlate of the OTR. Previous data showed that the perception of tilt of SVV depends not only on otolith information but also on somatosensory and visual signals (MacNeilage et al., 2007; Vingerhoets et al., 2009; Tarnutzer et al., 2010). It is well known that lesions of the eighth nerve as well as lesions of the vestibular nucleus cause an ipsiversive OTR (Halmagyi et al., 1979; Dieterich and Brandt, 1993), whereas lesions of the vestibular pathways in the pontomesencephalic brainstem such as the medial longitudinal fasciculus (MLF), its rostral interstitial nucleus (riMLF), and the interstitial nucleus of Cajal (INC) cause contraversive roll-tilts (Dieterich and Brandt, 1993; Brandt and Dieterich, 1994). Unilateral internuclear ophthalmoplegia was found to be associated with contraversive roll-tilts of SVV. This indicated that otolith pathways append the MLF (Zwergal et al., 2008a). Data testing 14 patients with pontomesencephalic stroke

\footnotetext{
Received Feb. 17, 2012; revised June 4, 2012; accepted June 12, 2012.

Author contributions: B.B. designed research; B.B., J.W., C.H., and C.G. performed research; B.B., F.T., and M.D. analyzed data; B.B., F.T., and M.D. wrote the paper.

This work was supported by the Deutsche Forschungsgemeinschaft (BA 4097/1-1, to B.B.). We thank Anja Schröer for data assessment.

The authors report no financial conflicts of interest.

Correspondence should be addressed to Dr. Bernhard Baier, Department of Neurology, University of Mainz, Langenbeckstrasse 1, 55131 Mainz, Germany. E-mail: baierb@uni-mainz.de.

DOI:10.1523/JNEUROSCI.0770-12.2012

Copyright $\odot 2012$ the authors $\quad 0270-6474 / 12 / 3214854-05 \$ 15.00 / 0$
}

found an association between an ipsiversive roll-tilt of SVV and an affection of the medial lemniscus [ML; ipsilateral vestibulothalamic tract (IVTT)]. This suggests the presence of an ipsilateral graviceptive pathway within the ML that connects the vestibular nuclei with the thalamus (Zwergal et al., 2008b).

Since previous data were not based on statistics (Dieterich and Brandt, 1993; Brandt and Dieterich, 1994; Zwergal et al., 2008b), we applied statistical voxelwise lesion-behavior mapping (VLBM) analysis to a sample of 79 patients with unilateral brainstem infarctions. This approach allows, for the first time, precise lesion localization and the determination of a statistical association between the degree of SVV roll-tilt and the voxels affected (Rorden et al., 2009). Moreover, the present approach has the potential advantage of being able to relate lesions directly to behavioral performance.

\section{Materials and Methods}

Subjects. Seventy-nine patients with magnetic resonance imaging (MRI)documented unilateral acute brainstem infarctions participated in the study [mean age, 67 years; standard deviation (SD), 13 years; 55 male patients (70\%)]. Forty-six patients (58\%) had left-side lesions and 33 patients (42\%) right-side lesions. The mean time between stroke and testing was $6 \mathrm{~d}(\mathrm{SD}, 3.0 \mathrm{~d})$ (Table 1). Patients in whom MRI scans revealed no obvious lesion were not included. The subjects gave their informed consent to participate in the study, which was approved by the local ethics committee.

Measurements of the SVV. Roll-tilts of the SVV in this setup-sitting upright in front of a spherical dome-are primarily a function of spatial perception due to an acute vestibular otolith dysfunction (Dieterich and Brandt, 1993). They are not simply the measure or sensory consequence of the ocular torsion (OT), which has to be performed by a red glass 
Table 1. Demographic and clinical data of all patients with contraversive and ipsiversive tilt of SVV

\begin{tabular}{lll}
\hline & Contraversive tilt of SVV & Ipsiversive tilt of SVV \\
\hline Number & 39 & 40 \\
Sex & $(12 \mathrm{f}, 27 \mathrm{~m})$ & $(12 \mathrm{f}, 28 \mathrm{~m})$ \\
Aetiology & 39 ischemic infarcts & 40 ischemic infarcts \\
Somatosensory deficit (touch) (\% present) & $11 \%$ & $24 \%$ \\
Hemiparesis (\% present) & $17 \%$ & $15 \%$ \\
Hemiataxia (\% present) & $25 \%$ & $27 \%$ \\
Diplopia (\% present) & $17 \%$ & $14 \%$ \\
Head tilt (\% present) & $9 \%$ & $8 \%$ \\
Binocular tilt of SVV (in degrees) [mean (SD) $]$ & $4.2^{\circ}(4.4)$ & $4.5^{\circ}(5.1)$ \\
Age (years) [mean (SD)] & $68^{\circ}(13)$ & $65^{\circ}(11.9)$ \\
TSL-clinical examination (days) [mean (SD) $]$ & $6.4^{\circ}(3.3)$ & $6.3^{\circ}(2.8)$ \\
\hline
\end{tabular}

$\mathrm{m}$, male; f, female; TSL, time since lesion.

directly in front of each eye (Bixenman and von Noorden, 1982). Here, patients sat in an upright position looking into a hemispheric dome at a distance of $\sim 1 \mathrm{~m}$ with the chin fixed at a chin rest. The patient was continuously urged by the orthoptic assistant to sit in an upright position to minimize the influence of head movements. The surface of the dome was covered with a random pattern of dots containing no clues as to visual gravitational orientation. The patients had to adjust the target disk (rod) at the center of the dome to the vertical by means of a joystick. Static SVV was determined by means of seven adjustments from a random offset position with the hemispheric dome stationary under binocular viewing conditions. Under these conditions, the normal range ( $\pm 2 \mathrm{SD}$ ) of the SVV is $\pm 2.5^{\circ}$ (Dichgans et al., 1972). A mean of $>2.5^{\circ}$ of the seven measurements of the static SVV determined binocularly was considered a criterion of a pathological roll-tilt of static SVV (Dieterich and Brandt, 1993). In case of diplopia, patients wore an eye patch and the adjustments were performed monocularly.

Fundus photographs. Fundus photographs for measurements of tonic OT as a sign of vestibular dysfunction were made using a scanning laser ophthalmoscope. OT, in degrees, was defined as the mean of four to six fundus photographs for each eye. The position of the eye in the roll plane was determined as the angle between a straight line through papilla and macula and the horizontal line. With this method, "normal" range of OT was defined as a range of $-1-11^{\circ}$ for the right eye and $0-11.5^{\circ}$ for the left eye (Dieterich and Brandt, 1993).

Skew deviation. Measurements of vertical divergence (hypertropia/hypotropia) of the eyes were determined as described previously with the head upright, first by red glass testing and second by using vertical orientated prisms (Dieterich and Brandt, 1993).

Imaging and lesion analysis. All patients had circumscribed brainstem lesions due to ischemic stroke demonstrated by MRI ( $1.5 \mathrm{~T}$ scanner Vision; Siemens). Thin-slice MRI ( $3 \mathrm{~mm}$; voxel size $\left.0.5 \times 0.5 \times 3 \mathrm{~mm}^{3}\right)$ T2-weighted fluid-attenuated inversion-recovery sequences were used to verify the brain lesions by diffusion-weighted images. The mean time between stroke onset and MRI imaging used for the present analyses was $4 \mathrm{~d}$ (SD, $2.5 \mathrm{~d})$.

The MRI dataset was first prepared using the isolation algorithm of the SUIT toolbox (Diedrichsen, 2006; Diedrichsen et al., 2009). Afterward, the normalization algorithm provided by SPM5 and the SUIT toolbox implemented in SPM5 were applied (Diedrichsen, 2006; Diedrichsen et al., 2009). For the normalization procedure, the isolation map was used as a mask based on the SPM interface. The lesions were delineated directly on the individual normalized MRI scans with MRIcron software (Rorden et al., 2009; http://www.mccauslandcenter.sc.edu/mricro/mricron/ install.html). Finally, the extent of the lesion shapes was compared with the original MRI dataset by a second neurologist.

To evaluate the relationship between lesion location and SVV roll-tilt, a VLBM analysis was performed by using the $t$ test statistic implemented in the MRIcron (Rorden et al., 2009). This algorithm uses SVV deviation as a continuous, dependent variable (and not a dichotomous variable) and separates, on the voxel level, those subjects that have a lesion of that voxel versus subjects who do not show a lesion of that specific voxel. Whereas patients with right- and with left-side lesions were examined together, i.e., left-side lesions were flipped to the right side, patients with contraversive and ipsiversive roll-tilt of the SVV were examined separately (Brandt and Dieterich, 1994). This approach is based on our a priori hypothesis that different regions are involved in contraversive and ipsiversive roll-tilt of SVV (Halmagyi et al., 1979; Dieterich and Brandt, 1993; Brandt and Dieterich, 1994). A subgroup analysis applying a qualitative subtraction method ( $n=14$ patients) tested whether patients with anterior paramedian pontomesencephalic infarctions and ipsiversive tilt of the SVV showed an association between tilt of the SVV and a possible IVTT (Zwergal et al., 2008b). To analyze whether patients with pathological OT differ with respect to their lesion location from patients with OT in the normal range, an additional subgroup analysis was conducted by applying a subtraction analysis. Subtraction analysis reflects the relative frequency of damage. Multiple comparisons were checked by using a false discovery rate (FDR) correction. All results presented below survived a 5\% FDR cutoff threshold. To identify the various structures affected, the lesions were compared with those in the brainstem atlas of Duvernoy (1995) and the stereotactic atlas of Schaltenbrand and Wahren (1977). Since the INC and the riMLF cannot be identified in MRI, neighboring reference structures were used, such as the oculomotor and trochlear nucleus or red nucleus (Helmchen et al., 2002). In addition, due to the lack of probabilistic maps in the brainstem, the identification of the INC and riMLF occurred with the help of the data reported by BüttnerEnnever et al. (1982) and Horn et al. (2000). Slices correspond to coordinates in MNI space.

\section{Results}

\section{Behavioral data}

Thirty-nine of all the patients presented with a contraversive rolltilt of the SVV (49\%) [mean, $4.2^{\circ}$ (absolute tilt of SVV); SD, $4.4^{\circ}$ ]; 40 of the patients (51\%) showed an ipsiversive roll-tilt of the SVV [mean, $4.5^{\circ}$ (absolute tilt of SVV); SD, $5.1^{\circ}$ ]. No difference was found between the two patient groups as to the extent of SVV deviation (unpaired $t=0.328 ; p=0.744$ ). The mean SD within subjects was $0.96^{\circ}$ for the patients with contralesional roll-tilt of SVV and $0.87^{\circ}$ for the patients with ipsilesional tilt.

If the criterion of a pathological roll-tilt of SVV of $>2.5^{\circ}$ was applied to our data (Dieterich and Brandt, 1993; Brandt and Dieterich, 1994), 42 of the 79 patients (53\%) showed pathological tilts. Nineteen of the 39 patients with contraversive roll-tilt of the SVV (49\%) were found to have pathological tilts, while 23 of the 40 patients with ipsiversive roll-tilt of the SVV (58\%) showed pathological tilts.

OT was measured in 61 of the 79 patients (77\%). Seven of the 29 patients with contraversive roll-tilt of SVV tested for OT presented with a pathological binocular OT (24\%) [mean tilt angle of the right eye of all patients with contraversive SVV tested for $\mathrm{OT}, 7^{\circ}\left(\mathrm{SD} 6^{\circ}\right)$; and of the left eye, $\left.9^{\circ}\left(\mathrm{SD} 6^{\circ}\right)\right]$, and four with ipsiversive roll-tilts presented with pathological OT (13\%) [mean tilt angle of the right eye, $6^{\circ}\left(\mathrm{SD} 5^{\circ}\right)$; and of the left eye, $8^{\circ}$ (SD $\left.5^{\circ}\right)$ ]. A complete OTR, i.e., head tilt, OT, and skew deviation was seen in seven of the 61 patients tested for OT (11\%). With regard to the somatosensory system (touch), no difference was seen in the extent of roll-tilt of SVV between patients with and without somatosensory deficits (unpaired $t$ test, $p=0.403$ ). No difference of the extent of roll-tilt of SVV was obtained between patients with hemiataxia, hemiparesis, head tilt, and diplopia and patients without these deficits (unpaired $t$ test, $p>0.05$ ).

\section{Anatomical data}

Figure $1 A$ illustrates lesion overlay plots of all patients. Figure $1 B$ shows the VLBM analysis for the contraversive roll-tilt of the SVV in patients. The superior cerebellar peduncle (SCP; brachium conjunctivum; $x=2, y=-23, z=-9$ ), the area of the oculomotor nucleus $(x=1, y=-25, z=-6)$, the riMLF $(x=3, y=$ $-29, z=-6)$, and the adjacent INC $(x=3, y=-31, z=-6)$ 
were significantly associated with contraversive roll-tilt of the SVV. Figure $1 C$ presents the VLBM data for the ipsiversive tilt. The MLF $(x=4, y=-41, z=-57)$ and the medial vestibular nucleus $(x=5$, $y=-41, z=-45)$ were found to be significantly associated with an ipsiversive deviation of the SVV. With regard to the subgroup analysis, no significant voxel was found between the anterior paramedian pontomesencephalic lesions of the 14 patients and ipsiversive roll-tilt of the SVV. Therefore, an additional subtraction analysis of the patients with pathological ipsiversive roll-tilt of the SVV $(n=8$; tilt of SVV $>2.5^{\circ}$ ) versus no roll-tilt of the SVV ( $n=6$; tilt of SVV $<2.5^{\circ}$ ) was conducted. This analysis indicated that small parts of the ML were affected in three patients with ipsiversive roll-tilt of the SVV $(x=7, y=-31, z=-28$; Fig. $1 D)$. The subgroup analysis of the seven patients with contraversive tilt of SVV and pathological OT versus those without OT revealed that voxels affecting regions such as the oculomotor nucleus $(x=4, y=-27$, $z=-7)$ and adjacent regions such as the $\operatorname{riMLF}(x=2, y=-30, z=-4), \operatorname{INC}(x=$ $1, y=-29, z=-4), \operatorname{MLF}(x=3, y=-32$, $z=-6)$, and the SCP $(x=4, y=-19, z=$ $-5)$ were damaged $29 \%$ more frequently in patients with than without pathological OT (Fig. 2A). The subtraction analysis of the four patients with ipsiversive tilt and pathological OT versus normal OT showed that the voxels affecting regions such as the vestibular nucleus $(x=7, y=-43, z=-48)$ and adjacent inferior cerebellar peduncle $(x=9$, $y=-42, z=-48$ ) were damaged $50 \%$ more frequently in patients with pathological OT than without (Fig. $2 B$ ).

To determine whether there exist a relationship between the variance within subjects and specific anatomical regions, we conducted an additional VLBM analysis. As seen in Figure 2, $C$ and $D$, no significant voxels could be obtained. This indicates that the variance within subjects is not explained by the affection of certain anatomical structures.
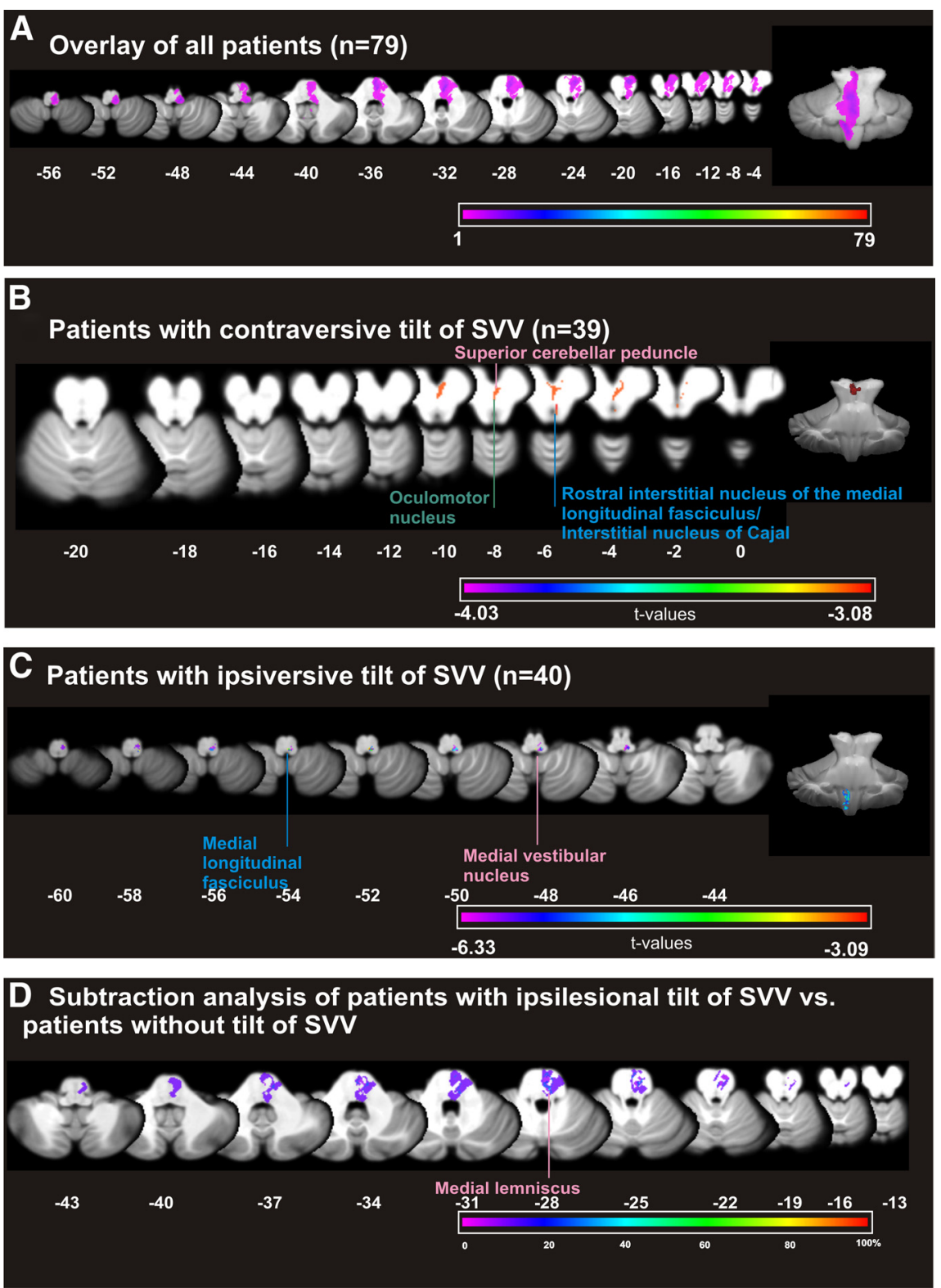

Figure 1. A, Simple overlay of all patients $(n=79)$; images of left-side lesions were flipped to the right side. The number of overlapping lesions is illustrated by different colors that code increasing frequencies from violet $(n=1)$ to red $(n=79)$. B, C, VLBM analysis comparing the patients with contraversive tilt of SVV $(n=39)(B)$ and the patients with ipsiversive tilt $(n=40)$ with respect to absolute SVV tilt ( $t$ test statistics) (C). All voxels that survived a correction for multiple comparisons using a $5 \%$ FDR cutoff threshold are shown. $\boldsymbol{D}$, Subtraction analysis of patients with lesions that affect the anteromedial pontomesencephalic region with tilt (tilt, $>2.5^{\circ}$ ) versus patients without tilt of the SVV. The percentage of overlapping lesions in the patients with tilt after subtraction of the controls is illustrated by different colors coding increasing frequencies, from violet $(0 \%)$ to dark red (100\%). This reflects the relative frequency of damage, i.e., the corresponding percentage of patients with tilt who are more frequently affected than in the control group.

\section{Discussion}

The present study verified that roll-tilt of the SVV is a sensitive brainstem sign (Dieterich and Brandt, 1993). Pathological SVV tilts occurred in $53 \%$ of the patients, whereas OT was found in $18 \%$ and complete OTR in $11 \%$. It was statistically confirmed for the first time that at the pontomedullary level, lesions of the MLF or the medial vestibular nucleus are associated with ipsiversive roll-tilt of the SVV. Contraversive roll-tilts are associated with lesions that affect the pontomesencephalic structures such as the SCP, the area of the oculomotor nucleus, or the riMLF and the INC. Thus, these structures seem to be directly involved in verti- cality perception within a multisensory system in which graviceptive pathways play a dominant role. In other words, since previous data showed strong evidence that graviceptive otolith signals subserve vestibular function (Halmagyi et al., 1979; Dieterich and Brandt, 1993; Tarnutzer et al., 2010), these structures are part of a vestibular graviceptive otolith brainstem pathway.

While otolith information is important for determining head orientation, somatosensory and visual information processing is also relevant (Yardley, 1990; MacNeilage et al., 2007; Tarnutzer et al., 2009; Vingerhoets et al., 2009). Previous data investigating the influence of the peripheral visual frame and lateral body tilt on 


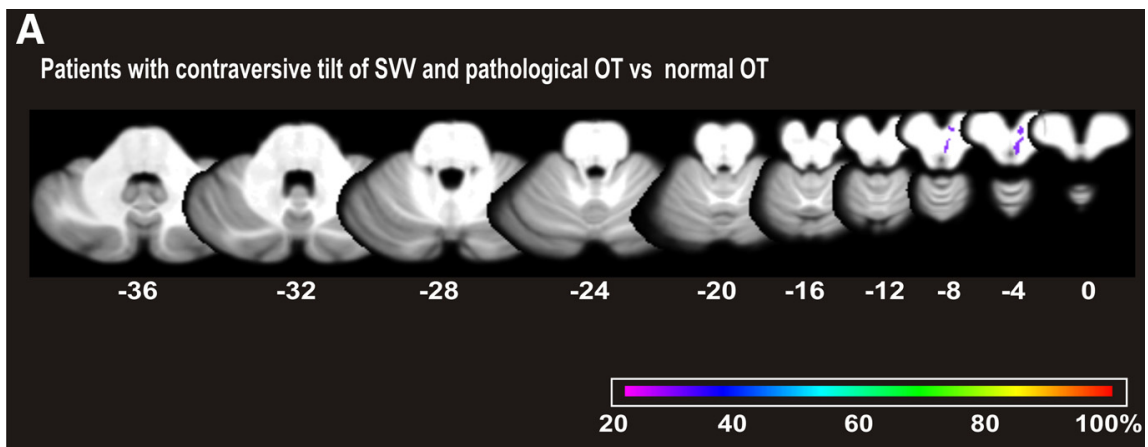

B

Patients with ipsiversive tilt of SVV and pathological OT vs normal OT

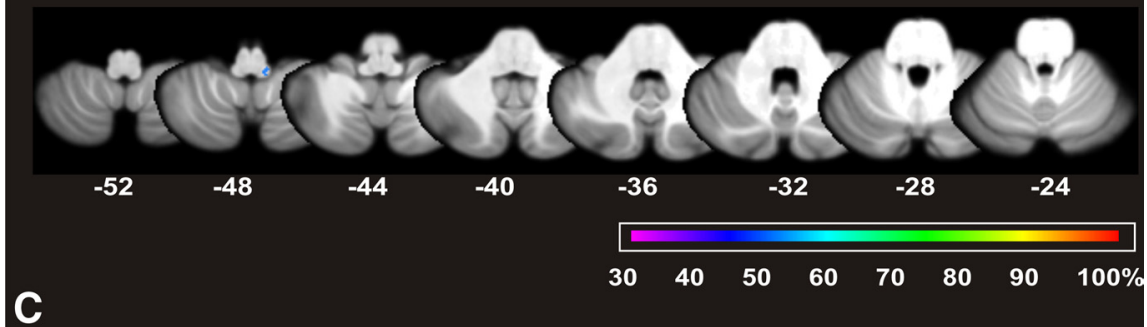

VLBM analysis of standard deviation within subjects of patients with contralesional tilt of SW (uncorrected)

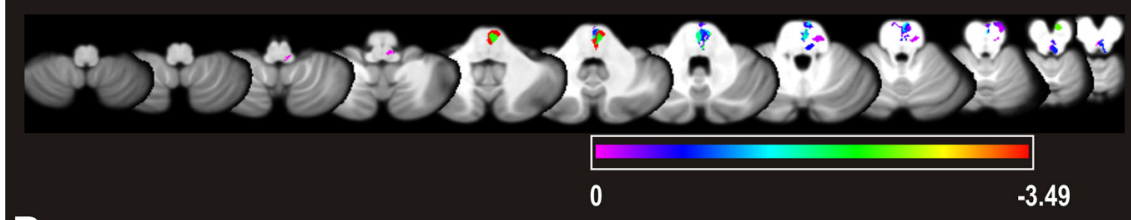

D

VLBM analysis of standard deviation within subjects of patients with ipsilesional tilt of SVV (uncorrected)

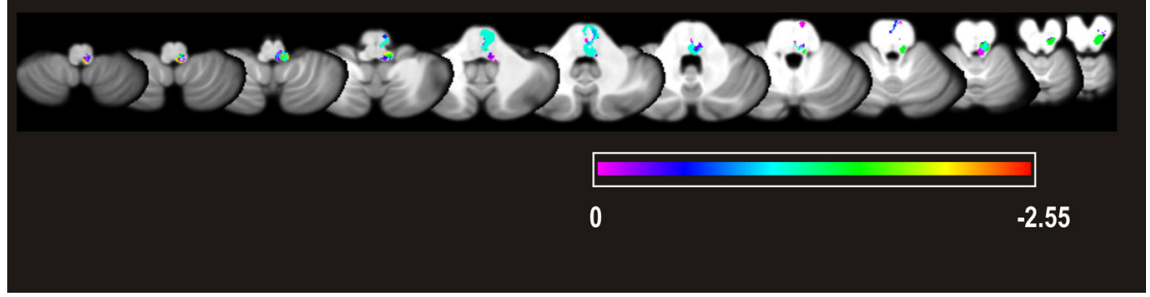

Figure 2. A, Subtraction analysis of patients with contraversive roll-tilts of SVV and pathological OT versus no 0 T. B, Subtraction analysis of patients with ipsiversive roll-tilts of SVV and pathological $0 T$ versus no 0 T. C, D, Uncorrected data including the standard deviation within subjects as continuous variable for the patients with contralesional $(\boldsymbol{C})$ and ipsilesional $(\boldsymbol{D})$ tilt of SVV.

the perception of the SVV concluded that visual vertical perception does not exclusively depend on vestibular signal processing but also on visual and egocentric signals (Vingerhoets et al., 2009). Another study (Tarnutzer et al., 2010) tested the contributions of vestibular and extravestibular signals to gravity perception, indicating that verticality perception depends mainly on vestibular signal processing. This is in line with our data showing no difference with regard to the extent of tilt of SVV observed between patients with somatosensory deficits and those without such deficits. However, previous data (MacNeilage et al., 2007; Angelaki and Cullen, 2008; Tarnutzer et al., 2009; Vingerhoets et al., 2009) undoubtedly suggest that vertical perception is based on multisensory integration of signal input, such as visual and somatosensory signals within regions such as the temporal and insular cortex, oculomotor nuclei, spinal cord, rostral fastigial nuclei, and thalamus (Angelaki and Yakusheva, 2009; Baier et al., 2012). With regard to oculomotor function in otolith pathways, one might assume that they compensate deficits in otolith signal processing. Therefore, it seems that tilt of SVV is predominantly provided by a multisensory reference frame (Tarnutzer et al., 2010) in which vestibular signal processing modulates visually and somatosensory biases in the perception of tilt of SVV. Taking into account that verticality perception is multimodal, even in the absence of a systematic somatosensory effect in the present study, somatosensory deficits might affect the fine-tuning of verticality perception (Bronstein et al., 1996).

In contrast to an earlier study (Dieterich and Brandt, 1993), the frequency of the pathological roll-tilt of the SVV was $53 \%$ compared with $94 \%$ in a sample of 111 patients with brainstem infarctions. This discrepancy, however, could be due to different lesion sizes, as our MRI techniques document much smaller lesions than those of earlier studies, or different time intervals between stroke onset and testing (Dieterich and Brandt, 1993).

Zwergal et al. (2008b) reported that an isolated ipsilateral graviceptive pathway between the vestibular nuclei and the posterolateral thalamus involved the medial part of the ML, the so-called IVTT. Lesions of this pathway might lead to an ipsiversive roll-tilt of the SVV. Our data did not identify any statistical involvement of the ML with ipsiversive roll-tilt of the SVV, which would be only a qualitative involvement. One reason might be that previous data were solely qualitative and their mapping was neither statistically based nor did they include a control group to perform a subtraction analysis showing the contrast of the lesions between patients with roll-tilt of SVV versus patients without tilt (Zwergal et al., 2008b). Alternatively, it might also be the case that in patients with lesions at the pontomesencephalic level that affect both structures, i.e., the ML causing an ipsilateral roll-tilt as well as the MLF leading to contraversive tilt, the two structures might compensate for each other. Thus, no significant anatomical association for contraversive roll-tilt of the SVV was obtained. Therefore, additional studies might find statistical support for the findings of an IVTT (Zwergal et al., 2008b).

The present study also disclosed for the first time that the SCP was involved in otolith dysfunction. This seems to represent a main structural connection to the cerebellar vestibular nuclei dealing with otolith signals. Support is thus given for the interaction with cerebellar vestibular otolith signal processing, which was recently investigated in more detail for the signs of an OTR in patients with acute unilateral cerebellar infarctions (Baier et al., 2008). SVV roll-tilts occurred in up to $84 \%$, more frequently to the contralateral (58\%) than the ipsilateral (26\%) side (Baier and Dieterich, 2009). The deviations in the acute phase were less in these cerebellar lesions $\left(5.8^{\circ}\right)$ and highest in patients with medullary brainstem infarctions $\left(9.8^{\circ}\right)$ (Cnyrim et al., 2007). The pathway from the brainstem to the cerebellar vermis seems to travel via the cerebellar peduncles-as also shown here- and the dentate nucleus to the pyramid of vermis, 
nodulus, and uvula, as well as the flocculus and tonsil (Baier et al., 2008). The involvement of cerebellar structures in vestibular signal processing in general and gravitational responses in particular is also shown by multiple lesion/stimulation experiments (for review, see Angelaki and Yakusheva, 2009). In detail, it was shown in former times that Purkinje cells of the uvula project to the vestibular nuclei (Shojaku et al., 1987).

Current data on the involvement of the oculomotor nucleus and its surrounding structures such as the INC and the riMLF are in line with the pathways and structures that mediate information of the vestibular end organ to the corresponding eye muscles via the vestibulo-ocular reflex (VOR). During eye, head, and body movements in the roll plane, torsional movements have to be elicited. They require, for example, the activation of oblique eye muscles mediated by the oculomotor and trochlear nuclei. Accordingly, unilateral lesions along the VOR pathways result not only in roll-tilts of the SVV but also in ocular torsion, caused by the deficit of activation (Dieterich and Brandt, 1993; Brandt and Dieterich, 1994). The INC and the riMLF are considered to be the neural integrator for torsional and vertical eye position and head posture as well as the vertical-torsional saccade generator (Crawford, 1994; Farshadmanesh et al., 2007; Kremmyda et al., 2011). Our data support the notion of their involvement in the pathological roll-tilt of the SVV due to otolithic signal inputs to the INC and the riMLF from the contralateral vestibular nucleus. Furthermore, contraversive rolltilts of the SVV caused by midbrain lesions are in line with the assumption of crossed otolith pathways between the vestibular nucleus and the INC/riMLF (Dieterich and Brandt, 1993). Thus, both structures - the INC and the riMLF — might be nodes that integrate gaze coordinate signals (Klier and Crawford, 2003) as well as otolith signals in one vestibulo-spatial coordinate frame.

With regard to OT, our data confirm that for OT as well as for tilt of SVV, similar regions such as the vestibular nucleus, the MLF, the inferior and superior peduncles, and the oculomotor nucleus were affected.

Since no difference in the extent of roll-tilt of SVV was obtained between patients showing clinical deficits such as hemiparesis, somatosensory deficits, head tilt, or hemiataxia and patients without such deficits, we argue that these deficits do not present possible confounding variables leading to false abnormal roll-tilt of SVV.

In summary, this is the first study using new statistical lesionbased methods on the brainstem level to compare anatomical landmarks with behavioral data on the SVV. Our data support the notion of otolith graviceptive pathways modulating vertical perception within a multisensory reference frame, indicating that lesions at the pontomesencephalic level lead to contraversive rolltilt, whereas lesions at the pontomedullary regions are associated with ipsiversive roll-tilt of the SVV.

The present study had certain limitations. Structural MRI scans might not necessarily show the full functional extent of a lesion. Areas that appear structurally intact in anatomical scans may not necessarily be functioning normally due to an abnormal perfusion.

\section{References}

Angelaki DE, Cullen KE (2008) Vestibular system: the many facets of a multimodal sense. Annu Rev Neurosci 31:125-150.

Angelaki DE, Yakusheva TA (2009) How vestibular neurons solve the tilt/ translation ambiguity. Comparison of brainstem, cerebellum, and thalamus. Ann NY Acad Sci 1164:19-28.

Baier B, Dieterich M (2009) Ocular tilt reaction: a clinical sign of cerebellar infarctions? Neurology 72:572-573.

Baier B, Bense S, Dieterich M (2008) Are signs of ocular tilt reaction in patients with cerebellar lesions mediated by the dentate nucleus? Brain 131:1445-1454.

Baier B, Suchan J, Karnath HO, Dieterich M (2012) Neural correlates of disturbed perception of verticality. Neurology 78:728-735.

Bixenman WW, von Noorden GK (1982) Apparent foveal displacement in normal subjects and in cyclotropia. Ophthalmology 89:58-62.

Brandt T, Dieterich M (1994) Vestibular syndromes in the roll plane: topographic diagnosis from brainstem to cortex. Ann Neurol 36:337-347.

Bronstein AM, Yardley L, Moore AP, Cleeves L (1996) Visually and posturally mediated tilt illusion in Parkinson's disease and in labyrinthine defective subjects. Neurology 47:651-656.

Büttner-Ennever JA, Büttner U, Cohen B, Baumgartner G (1982) Vertical gaze paralysis and the rostral interstitial nucleus of the medial longitudinal fasciculus. Brain 105:125-149.

Cnyrim CD, Rettinger N, Mansmann U, Brandt T, Strupp M (2007) Central compensation of deviated subjective visual vertical in Wallenberg's syndrome. J Neurol Neurosurg Psychiatry 78:527-528.

Crawford JD (1994) The oculomotor neural integrator uses a behaviorrelated coordinate system. J Neurosci 14:6911-6923.

Dichgans J, Held R, Young LR, Brandt T (1972) Moving visual scenes influence the apparent direction of gravity. Science 178:1217-1219.

Diedrichsen J (2006) A spatially unbiased atlas template of the human cerebellum. Neuroimage 33:127-138.

Diedrichsen J, Balsters JH, Flavell J, Cussans E, Ramnani N (2009) A probabilistic MR atlas of the human cerebellum. Neuroimage 46:39-46.

Dieterich M, Brandt T (1993) Ocular torsion and tilt of subjective visual vertical are sensitive brainstem signs. Ann Neurol 33:292-299.

Duvernoy HM (1995) The human brain stem and cerebellum. Springer: Wien. Farshadmanesh F, Klier EM, Chang P, Wang H, Crawford JD (2007) Threedimensional eye-head coordination after injection of muscimol into the interstitial nucleus of Cajal (INC). J Neurophysiol 97:2322-2338.

Halmagyi GM, Gresty MA, Gibson WP (1979) Ocular tilt reaction with peripheral vestibular lesion. Ann Neurol 6:80-83.

Helmchen C, Rambold H, Kempermann U, Büttner-Ennever JA, Büttner U (2002) Localizing value of torsional nystagmus in small midbrain lesions. Neurology 59:1956-1964.

Horn AK, Büttner-Ennever JA, Gayde M, Messoudi A (2000) Neuroanatomical identification of mesencephalic premotor neurons coordinating eyelid with upgaze in the monkey and man. J Comp Neurol 420:19-34.

Klier EM, Crawford JD (2003) Neural control of three-dimensional eye and head posture. Ann N Y Acad Sci 1004:122-131.

Kremmyda O, Glasauer S, Guerrasio L, Büttner U (2011) Effects of unilateral midbrain lesions on gaze (eye and head) movements. Ann N Y Acad Sci 1233:71-77.

MacNeilage PR, Banks MS, Berger DR, Bülthoff HH (2007) A Bayesian model of the disambiguation of gravitoinertial force by visual cues. Exp Brain Res 179:263-290.

Rorden C, Fridriksson J, Karnath HO (2009) An evaluation of traditional and novel tools for lesion behavior mapping. Neuroimage 44:1355-1362.

Schaltenbrand G, Wahren W (1977) Atlas for stereotaxy of the human brain. Georg Thieme: Stuttgart.

Shojaku H, Sato Y, Ikarashi K, Kawasaki T (1987) Topographical distribution of Purkinje cells in the uvula and the nodulus projecting to the vestibular nuclei in cats. Brain Res 416:100-112.

Tarnutzer AA, Bockisch C, Straumann D, Olasagasti I (2009) Gravity dependence of subjective visual vertical variability. J Neurophysiol 102:1657-1671.

Tarnutzer AA, Bockisch CJ, Straumann D (2010) Roll-dependent modulation of the subjective visual vertical: contributions of head- and trunkbased signals. J Neurophysiol 103:934-941.

Vingerhoets RA, De Vrijer M, Van Gisbergen JA, Medendorp WP (2009) Fusion of visual and vestibular tilt cues in the perception of visual vertical. J Neurophysiol 101:1321-1333.

Yardley L (1990) Contribution of somatosensory information to perception of the visual vertical with body tilt and rotating visual field. Percept Psychophys 48:131-134.

Zwergal A, Cnyrim C, Arbusow V, Glaser M, Fesl G, Brandt T, Strupp M (2008a) Unilateral INO is associated with ocular tilt reaction in pontomesencephalic lesions: INO plus. Neurology 71:590-593.

Zwergal A, Büttner-Ennever J, Brandt T, Strupp M (2008b) An ipsilateral vestibulothalamic tract adjacent to the ML in humans. Brain 131:2928-2935. 